\title{
Quasi-Periodicities at Year Time Scales in Blazars
}

\author{
Stefano Covino ${ }^{1}$, Angela Sandrinelli ${ }^{1,2}$ and Aldo Treves ${ }^{2}$ \\ ${ }^{1}$ INAF / Brera Astronomical Observatory, via Bianchi 46, 23897, Merate (LC), Italy \\ ${ }^{2}$ Università degli Studi dell'Insubria, Via Valleggio 11, 22100 Como, Italy
}

\begin{abstract}
We examine the 2008-2016 gamma-ray and optical light curves of a number of bright Fermi blazars. In a fraction of them, the periodograms show possible evidence of quasiperiodicities related in the two bands. This coincidence strengthens their physical meaning. Comparing with results from the periodicity search of quasars, the presence of quasi-periodicities in blazars suggests that the basic condition for its observability is related to the relativistic jet in the observer direction, but the overall picture remains uncertain.
\end{abstract}

Keywords. BL Lacertae objects: general, methods: statistical

\section{Introduction}

Blazars are active galactic nuclei with a relativistic jet pointing in the observer direction. They are highly variable in all spectral bands (e.g. Falomo et al. 2014), and periodicities for these sorces have been hypothesized since the discovery of the first members of the class. Yet, no fully convincing case has been identified, with the possible exception of OJ 287, with a reported 12 year period (e.g. Sillanpää et al. 1988) that appears rather robust, but not uncontroversial (e.g. Hudec et al. 2013).

In recent years it has become also clear that blazars are the main constituents of the extragalactic $\gamma$-ray sky and the Fermi mission since its launch has monitored the entire celestial sphere every 3 hours. Therefore, $\gamma$-ray light curves of blazars are becoming easily available (Abdo et al. 2010), opening the possibility to look for periodicities in multiple and independent bands. In addition, robotic telescopes often developed for the monitorig of high-energy transients as gamma-ray bursts, are now operational since almost a decade, providing a large amount of homogeneous data that can fruitfully be analyzed for identifying long-term oscillations.

One of these small-size robotic facilities is the Rapid Eye Mount (REM, Zerbi et al. 2004; Covino et al. 2004) telescope (Fig. 1) located at the ESO premise of La Silla since 2004 and since then devoting a large fraction of the available observing time to blazar monitoring in the Southern hemisphere (Fig.2). Our team derived and analyzed the REM data for six BL Lac objects: PKS 0537-441, PKS 0735+17, OJ 287, PKS 2005489, PKS 2155-304, and W Comae, and of the flat spectrum radio quasar PKS 1510-089 (Sandrinelli et al. 2014a).

\section{Quasi Periodicities}

For some of the monitored sources, a search for periodicity has been carried out with optical, from REM and other facilities, and high-energy Fermi data (Sandrinelli et al. 2014b, 2016a,b). We recently also performed analogous analyses on three BL Lac objects in the Northen sky $(0716+714$, MRK 421 and BL Lac, Sandrinelli et al. 2017) and similar investigations have been proposed by other teams (e.g. Ackermann et al. 2015). Generally 


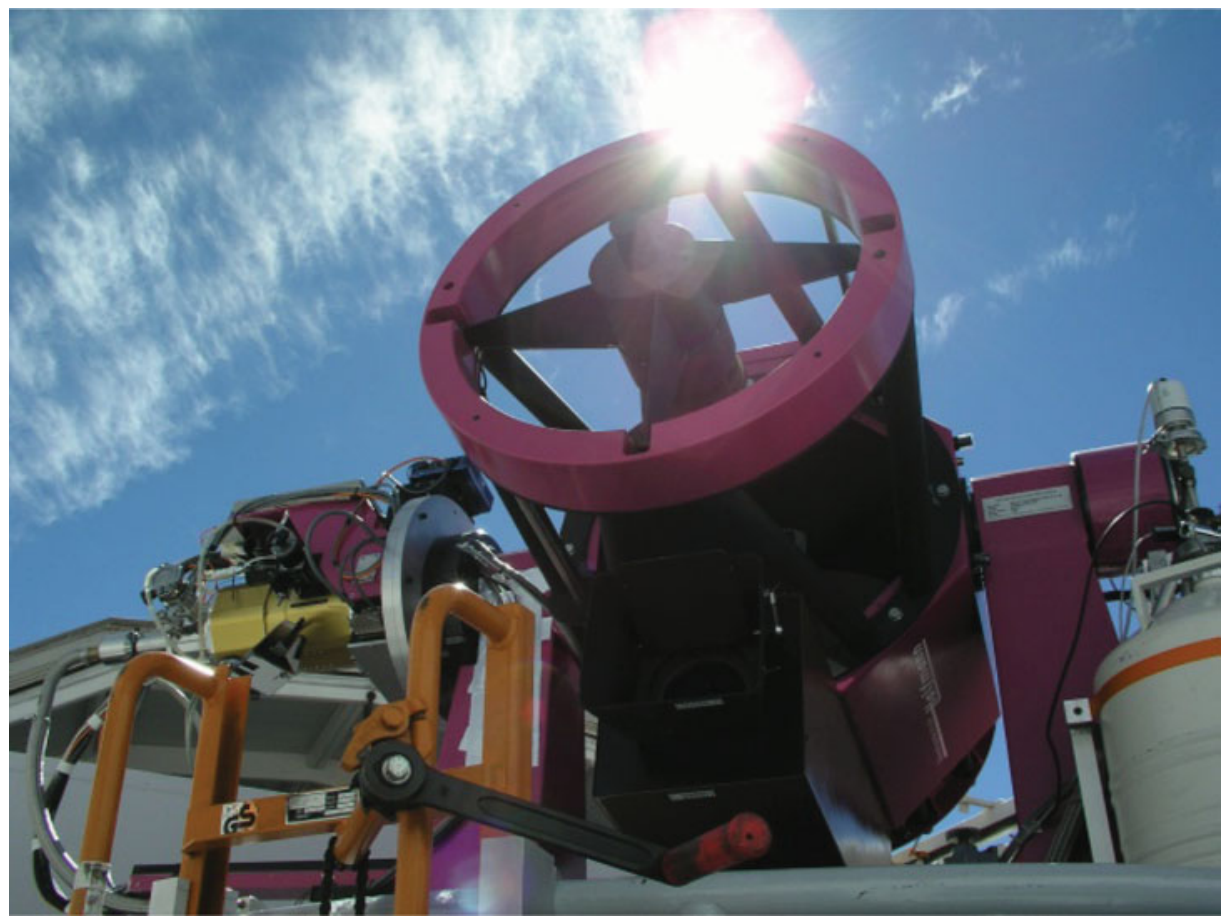

Figure 1. The REM telescope at the ESO facility of La Silla.

speaking, in a fair fraction of the analyzed sources, possible periodicities at year timescale have been singled out (Fig. 3).

The proposed quasi-periodicities correspond to peaks in the power density spectra that stand out of the local structured noise rather clearly. However, once a correction for the "trial-factors" due to the large number of frequencies analyzed is applied, the global significance of the periods turns out to be rather low. The whole subject is rather complex and widely discussed in the literature (e.g. Horne \& Baliunas 1986; Frescura et al. 2008; Süveges 2014; Süveges et al. 2015; Vaughan 2005, 2010), and a proper treatment of the statistical significance of the discussed quasi-periodicities will be subject of a forthcoming paper. At this stage it is enough to consider that, beyond any correct evaluation of the chance probabilities for these findings, we observe that in several cases there is a coincidence, within the errors, of the same periods in independent bands as the optical and gamma-rays. This suggests that there could be a physical meaning associated to these periods worth discussing in some detail.

\section{Discussion}

For a few sources low-significance periodicities simultaneously present in the optical and in the gamma-rays have been identified, enforcing their possible physical relevance. The convincing cases are PKS 2155-304 (Sandrinelli et al. 2014b), PG 1553+11 (Ackermann et al. 2015), PKS 0537-447 (Sandrinelli et al. 2016b) and BL Lac (Sandrinelli et al. 2017). These oscillations could be directly related to a real periodicity, as the orbital one of a system of two binary supermassive black holes, or indirectly as being due to an accretion disc/jet precession.

Blazar emissions are characterized by a chaotic component with superimposed a possible periodicity, which is therefore possibly hidden and difficult to single out if, as it is 


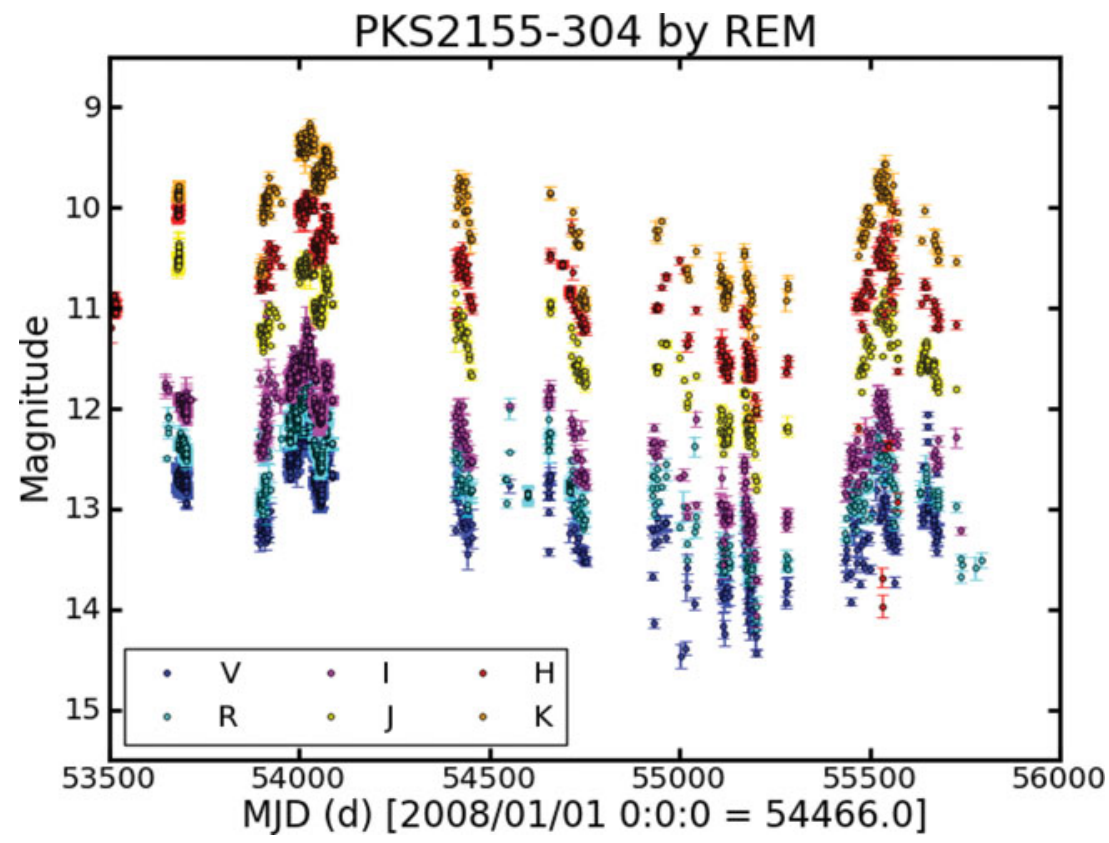

Figure 2. Multicolor light-curve for PKS2155-304.

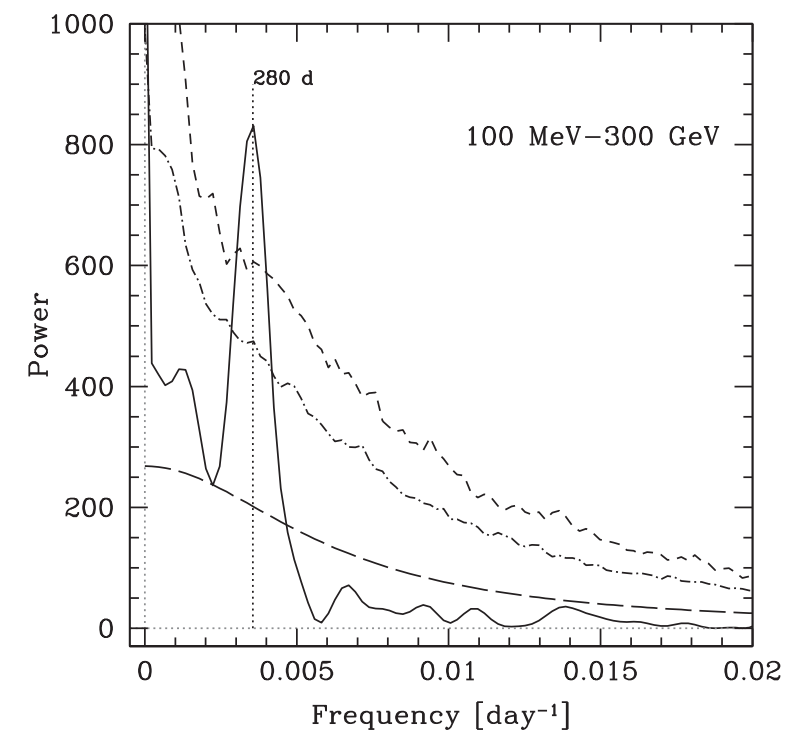

Figure 3. Power density spectrum for the Fermi gamma-ray data of an active phase of PKS 0537-441 (black line, from Sandrinelli et al. 2016b). A possible periodicity at $T \sim 280$ days is indicated. $95 \%$ and $99 \%$ significance curves derived from the local noise model (dashed line) are also shown.

typically the case, the data are unevenly sampled and the time-series have of course a finite length. Furthermore, the fact that optical observation campaigns on a blazar are basically activated in case of a high state of the source introduces another complicating factor. 
Recently, year-like periodicities have been searched for quasars starting from various surveys, detecting several positive candidate cases among hundred thousands objects (Charisi et al. 2016, and references therein). Therefore, there could be a remarkable difference with respect to bright Fermi blazars in terms of fraction of quasi-periodicity detections with roughly a year time-scale. If this will be proved to be true it could be related with the main characteristic that distinguishes them from quasars, the presence of a relativistic jet pointing in the observer direction. The net effect would be to obviously amplify the visibility of the oscillations, since it can magnify the variability through known relativistic effects. Furthermore, as already mentioned, it is possible that the oscillations are due to jet instabilities, independent of the presence of a binary companion.

\section{References}

Abdo, A. A., Ackermann, M., Ajello, M., et al. 2010, ApJ, 722, 520

Ackermann, M., Ajello, M., Albert, A., et al. 2015, ApJ, 813, 41

Charisi, M., Bartos, I., Haiman, Z., et al. 2016, MNRAS, 463, 2145

Covino, S., Stefanon, M., Sciuto, G., et al. 2004, SPIE 5492, 1613

Falomo, R., Pian, E., \& Treves, A. 2014, ARAA, 22, 73

Frescura, F. A. M., Engelbrecht, C. A., \& Frank, B. S. 2008, MNRAS, 388, 1693

Horne, J. H. \& Baliunas, S. L. 1986, ApJ, 302, 757

Hudec, R., Bašta, M., Pihajoki, P., \& Valtonen, M. 2013, AשEA, 559, A20

Sandrinelli, A., Covino, S., \& Treves, A. 2014a, A\&A, 562, 79

Sandrinelli, A., Covino, S., \& Treves, A. 2014b, ApJ, 793, 1

Sandrinelli, A., Covino, S., \& Treves, A. 2016a, AJ, 151, 54

Sandrinelli, A., Covino, S., \& Treves, A. 2016b, ApJ, 820, 20

Sandrinelli, A., Covino, S., Treves, A., et al. 2017, A\&SA, in press (arXiv:1701.04454)

Sillanpää, A., Haarala, S., Valtonen, M. J., Sundelius, B., \& Byrd, G. G. 1988, ApJ, 325, 628

Süveges, M. 2014, MNRAS, 440, 2099

Süveges, M., Guy, L. P., Eyer, L., et al. 2015, MNRAS, 450, 2052

Vaughan, S. 2005, A\&A, 431, 391

Vaughan, S. 2010, MNRAS, 402, 307

Zerbi, F. M., Chincarini, G., Ghisellini, G., et al. 2004, SPIE, 5492, 1590 